\title{
A cubic equation of state for vapor-liquid equilibrium calculations of nonpolar and polar fluids
}

\author{
Huei-Tung Wang, Jung-Chin Tsai, Yan-Ping Chen * \\ Department of Chemical Engineering, National Taiwan University, Taipei, Taiwan \\ Received 1 January 1997; accepted 7 May 1997
}

\begin{abstract}
A cubic perturbed hard body (CPHB) equation of state (EOS) was developed and applied for vapor-liquid equilibrium (VLE) calculations in this study. The repulsive part of this EOS was investigated in our previous work. It is a simplified expression of hard body equation which yielded satisfactory agreement to the molecular simulation data of hard molecules from a single sphere to long chain fluids. An empirical attractive term was combined with the repulsive contribution, and the total EOS was written in a cubic form. This EOS has three parameters for each real pure fluid. Pure fluid parameters were regressed in this study for nonpolar and polar fluids. Satisfactory calculated results of the saturated properties of pure fluids were obtained. The accuracy of the CPHB EOS is better than that of other two- or three-parameter cubic MSRK, CCOR, PT or PRSV2 EOSs. VLE calculations on fluid mixtures were also investigated using the van der Waals mixing rules. The CPHB EOS again gave good results, which are comparable to those from other cubic EOSs. (C) 1997 Elsevier Science B.V.
\end{abstract}

Keywords: Equation of state; Cubic; Vapor-liquid equilibria; Method of calculation; Nonpolar and polar

\section{Introduction}

Equation of state (EOS) models, especially cubic type EOSs, have been extensively employed in phase equilibrium calculations for chemical process design. A cubic EOS comprises two parts due to the repulsive and attractive forces between molecules. Various applications of the simplified forms of the Carnahan-Starling hard sphere EOS ([1]) for the repulsive part of a cubic EOS have been presented in literature. Examples are the CCOR EOS ([2]) and the perturbed hard sphere EOS (e.g., $[3,4]$ ). For nonspherical hard cores, an extra parameter is needed to describe their shapes. For example, Boublik [5] used a nonspherical factor $\alpha$ for hard fused bodies in his improved scaled

\footnotetext{
${ }^{*}$ Corresponding author. Tel.: + 886-2366-1661; fax: +886-2362-3040; e-mail: ypchen@ccms.ntu.edu.tw
} 
particle theory (ISPT). Walsh and Gubbins [6] developed the modified thermodynamic perturbation theory (MTPT) of polymerization, which can suitably simulate the thermodynamic properties of hard chain molecules of various shapes ([7]). Chen et al. [8] used a simplified form of the Walsh-Gubbins equation as the repulsive part of an EOS. A non-spherical shape parameter was employed for hard molecules of various shapes and geometries. Compressibility factors of tangent and fused hard fluids from a single sphere to a hard chain of 201 tangent spheres were calculated, and the results are in good agreement with the computer simulation data from literature. Chen et al. [8] have used this repulsive equation and an empirical attractive part to develop a cubic perturbed hard body (CPHB) EOS. They have shown good calculation results for nonpolar fluids and their mixtures. In this study, we extended the CPHB EOS to calculations on both nonpolar and polar fluids. This EOS has a good theoretical basis for the repulsive part and can describe well the phase behavior of fluids with appropriate real fluid parameters. New EOS parameters were correlated and comparisons of the calculated results with other cubic type EOSs were presented.

\section{Development of the CPHB EOS}

Walsh and Gubbins [6] had developed their hard-body EOS, which expressed the compressibility factor as a function of the packing fraction and a nonspherical factor. Chen et al. [8] applied the Walsh-Gubbins EOS and simplified it into the following form:

$$
Z^{\text {rep }}=\frac{V+k_{1} b}{V-k_{2} b}
$$

where $b$ is the hard core volume and $b / V$ represents the packing fraction. $k_{1}$ and $k_{2}$ are two parameters which are functions of the shapes of hard molecules that vary from a single sphere to chainlike molecules. These $k$ parameters were determined by regression of molecular simulation data of hard fluids. According to our previous work, they were correlated as:

$$
\begin{aligned}
& k_{1}=4.8319 \alpha-1.5515 \\
& k_{2}=1.8177-0.1778 \alpha^{-1.3686}
\end{aligned}
$$

where $\alpha$ is a nonspherical factor that characterizes various shapes of molecules. For a single hard sphere, $\alpha$ is unity and Eq. (1) reduces to approximate the Carnahan-Starling EOS ([1]). Chen et al. [8] have shown that Eq. (1) represented well the compressibility factors for various nonspherical hard molecules with different $\alpha$ values. To complete the EOS, an empirical attractive term was added to Eq. (1):

$$
P=\frac{R T\left(1+k_{1} b / V\right)}{V-k_{2} b}-\frac{a}{V(V+c)}
$$

where $a, b$ and $c$ are EOS parameters that are determined from the critical properties of pure fluids. At the critical point, the CPHB EOS satisfies the following conditions:

$$
\begin{aligned}
& (\partial P / \partial V)_{\mathrm{c}}=\left(\partial^{2} P / \partial V^{2}\right)_{\mathrm{c}}=0 \\
& P_{\mathrm{c}} V_{\mathrm{c}} /\left(R T_{\mathrm{c}}\right)=\zeta_{\mathrm{c}}
\end{aligned}
$$


Applying the above criteria, the EOS parameters $a, b$ and $c$ were then determined by:

$$
\begin{aligned}
& a=\frac{\Omega_{a \mathrm{c}} R^{2} T_{\mathrm{c}}^{2}}{P_{\mathrm{c}}} F_{1}(T) \\
& b=\frac{\Omega_{b \mathrm{c}} R T_{\mathrm{c}}}{P_{\mathrm{c}}} F_{2}(T) \\
& c=\frac{\Omega_{\mathrm{cc}} R T_{\mathrm{c}}}{P_{\mathrm{c}}}
\end{aligned}
$$

Parameters $a$ and $b$ are treated as temperature-dependent and $F_{1}$ and $F_{2}$ are two functions of the reduced temperature. $\Omega_{a c}, \Omega_{b c}$ and $\Omega_{c c}$ were determined from the following equations:

$$
\begin{aligned}
& k_{2}^{3} \Omega_{b c}^{3}+\left(2 k_{1} k_{2}+2 k_{2}^{2}-3 k_{2}^{2} \zeta_{c}\right) \Omega_{b c}^{2}+\left(k_{1}+k_{2}-3 k_{1} \zeta_{c}-3 k_{2} \zeta_{c}+3 k_{2} \zeta_{c}^{2}\right) \Omega_{b c}-3 \zeta_{c}=0 \\
& \Omega_{c c}=1+k_{2} \Omega_{b c}-3 \zeta_{c} \\
& \Omega_{a c}=\frac{\zeta_{c}^{3}-k_{1} \Omega_{b c} \Omega_{c c}}{k_{2} \Omega_{b c}}
\end{aligned}
$$

Parameter $\zeta_{\mathrm{c}}$, the nonspherical factor $\alpha$, and the temperature-dependent functions $F_{1}$ and $F_{2}$ were determined for each pure fluid. These values were then correlated by generalized equations of pure fluid parameters. To evaluate the EOS parameters, the equal fugacity criterion on each isotherm of the saturation curve was applied in this study:

$$
f^{\mathrm{v}}=f^{\mathrm{L}}
$$

The fugacity coefficient expression of the CPHB EOS is:

$$
\ln \frac{f}{P}=Z-1-\ln Z+\frac{k_{1}+k_{2}}{k_{2}} \ln \frac{Z}{Z-k_{2} B}+\frac{A}{C} \ln \frac{Z}{Z+C}
$$

where

$$
\begin{aligned}
& A=P a /(R T)^{2} \\
& B=P b / R T \\
& C=P c / R T
\end{aligned}
$$

Using the experimental data of saturated vapor pressure and liquid molar volume, $\zeta_{c}, \alpha, F_{1}$ and $F_{2}$ values were regressed for each pure fluid. The following objective function was minimized in the regressions:

$$
Q=\frac{100}{N_{P}} \sum_{i}^{N P}\left|\frac{P_{i}^{\mathrm{cal}}-P_{i}^{\exp }}{P_{i}^{\exp }}\right|+\frac{100}{N_{V}} \sum_{j}^{N V}\left|\frac{V_{j}^{\mathrm{cal}}-V_{j}^{\exp }}{V_{j}^{\exp }}\right|
$$


This EOS was also applied for the VLE calculations of both nonpolar and polar fluid mixtures. In mixture calculations, the following mixing rules were used to determine the mixture parameters from the constituent components:

$$
\begin{aligned}
& a_{m}=\sum \sum x_{i} x_{j} \sqrt{a_{i} a_{j}}\left(1-k_{i j}\right) \\
& b_{m}=\sum x_{i} b_{i} \\
& c_{m}=\sum x_{i} c_{i} \\
& \alpha_{m}=\sum x_{i} \alpha_{i}
\end{aligned}
$$

The fugacity coefficient of component $i$ in a mixture is expressed as:

$$
\begin{aligned}
\ln \hat{\phi}_{i}= & \ln \frac{V}{V-k_{2} b}-\frac{a\left(\partial n c / \partial n_{i}\right)}{R T\left(c^{2}+c V\right)}+\frac{1}{R T}\left(\frac{1}{n c} \frac{\partial n^{2} a}{\partial n_{i}}-\frac{a}{c^{2}} \frac{\partial n c}{\partial n_{i}}\right) \ln \frac{V}{V+c} \\
& +\frac{\left(k_{1}+k_{2}\right)\left[k_{2}\left(\partial n b / \partial n_{i}\right)+n b\left(\partial k_{2} / \partial n_{i}\right)\right]}{k_{2} V-k_{2}^{2} b} \\
& -\ln Z+\frac{\left[\left(k_{1} k_{2}+n k_{2}\left(\partial k_{1} / \partial n_{i}\right)-n k_{1}\left(\partial k_{2} / \partial n_{i}\right)\right]\right.}{k_{2}^{2}} \ln \frac{V}{V-k_{2} b}
\end{aligned}
$$

where

$$
\begin{aligned}
& \frac{1}{n} \frac{\partial n^{2} a}{\partial n_{i}}=2 \sum_{j} x_{j} a_{j i} \\
& \frac{\partial n b}{\partial n_{i}}=b_{i} \\
& \frac{\partial n c}{\partial n_{i}}=c_{i} \\
& n \frac{\partial k_{1}}{\partial n_{i}}=4.8319\left(\alpha_{i}-\alpha\right) \\
& n \frac{\partial k_{2}}{\partial n_{i}}=0.2433 \alpha_{i}^{-2.3686}\left(\alpha_{i}-\alpha\right)
\end{aligned}
$$

For the VLE calculations of fluid mixtures, the fugacity of component $i$ in the equilibrium liquid and vapor phases are equal:

$$
\hat{f}_{i}^{\mathrm{V}}=\hat{f}_{i}^{\mathrm{L}}
$$


Table 1

Pure fluid parameters and comparison of pure fluid calculation results

\begin{tabular}{|c|c|c|c|c|c|c|c|c|c|c|}
\hline \multirow[t]{2}{*}{ Compound } & \multicolumn{2}{|c|}{ Temp. Range (K) } & \multicolumn{2}{|c|}{ No. of data pts. } & \multirow[t]{2}{*}{$C_{1}$} & \multirow[t]{2}{*}{$C_{2}$} & \multirow[t]{2}{*}{$C_{3}$} & \multicolumn{2}{|c|}{$\operatorname{AAD}(\%)$} & \multirow[t]{2}{*}{ Data ref } \\
\hline & $P^{\mathrm{V}}$ & $V^{\mathrm{L}}$ & $\overline{P^{v}}$ & $V^{\mathrm{L}}$ & & & & $\overline{P^{V}}$ & $\overline{V^{\mathrm{L}}}$ & \\
\hline Argon & $83-148$ & $94-148$ & 67 & 55 & 2.88480 & 1.29716 & 0.26406 & 1.79 & 3.56 & [30] \\
\hline Krypton & $116-208$ & $116-208$ & 47 & 46 & 2.61571 & 1.09862 & -0.02402 & 1.34 & 3.73 & [30] \\
\hline Oxygen & $74-152$ & $89-152$ & 80 & 65 & 2.10809 & 1.46570 & -0.71923 & 1.87 & 2.71 & [30] \\
\hline Nitrogen & $63-125$ & $68-125$ & 64 & 59 & 1.45165 & 1.61732 & -1.28579 & 1.30 & 2.76 & [30] \\
\hline Carbon dioxide & $217-302$ & $217-302$ & 42 & 42 & -0.56727 & 3.58918 & -4.71014 & 0.08 & 1.02 & [30] \\
\hline Sulfur dioxide & $203-428$ & $323-431$ & 31 & 20 & -1.10328 & 3.45813 & -4.38037 & 0.69 & 0.55 & [30] \\
\hline Hydrogen chloride & $159-240$ & $170-230$ & 11 & 8 & 1.05583 & 1.47471 & -0.93925 & 0.69 & 2.03 & [27] \\
\hline Ammonia & $200-406$ & $200-390$ & 22 & 20 & 0.55909 & 1.98982 & -1.80059 & 0.23 & 2.13 & [30] \\
\hline Water & $273-647$ & $273-647$ & 97 & 97 & 0.28111 & 2.18987 & -2.03823 & 0.17 & 3.11 & {$[30]$} \\
\hline Methane & $91-187$ & $111-185$ & 44 & 34 & 2.24945 & 1.25414 & -0.40022 & 1.73 & 2.5 & [29] \\
\hline Ethane & $163-300$ & $163-300$ & 29 & 29 & 1.15111 & 1.93724 & -1.59748 & 0.91 & 2.83 & [29] \\
\hline Propane & $173-363$ & $173-363$ & 31 & 31 & 0.26010 & 2.35833 & -2.60292 & 0.55 & 2.26 & [29] \\
\hline Butane & $217-392$ & $217-392$ & 28 & 28 & -0.68396 & 2.92231 & -3.55943 & 0.29 & 1.03 & [29] \\
\hline Isobutane & $187-401$ & $213-369$ & 34 & 25 & -0.51955 & 2.91328 & -3.66925 & 0.62 & 0.92 & [29] \\
\hline Pentane & $240-454$ & $240-454$ & 30 & 30 & -1.24179 & 3.31679 & -3.96418 & 0.08 & 0.57 & [29] \\
\hline 2-Methylbutane & $236-447$ & $236-447$ & 29 & 29 & -1.12959 & 3.15123 & -3.70518 & 0.24 & 0.97 & [29] \\
\hline 2,2-Dimethylpropane & $257-433$ & $261-429$ & 45 & 43 & -0.90309 & 3.00982 & -3.60081 & 0.10 & 1.11 & [29] \\
\hline Hexane & $267-492$ & $267-492$ & 31 & 31 & -1.12066 & 3.60780 & -3.62742 & 0.28 & 0.82 & [29] \\
\hline 2-Methylpentane & $289-497$ & $289-480$ & 25 & 23 & -1.15511 & 3.51122 & -3.53593 & 0.59 & 1.06 & [29] \\
\hline 3-Methylpentane & $283-504$ & $273-494$ & 43 & 43 & -1.19541 & 3.53845 & -3.82397 & 0.18 & 0.79 & [29] \\
\hline 2,2-Dimethylbutane & $236-488$ & $274-463$ & 41 & 31 & -1.18724 & 3.17705 & -3.50889 & 0.27 & 0.43 & [29] \\
\hline 2,3-Dimethylbutane & $259-499$ & $275-489$ & 45 & 40 & -1.20558 & 3.28965 & -3.56648 & 0.33 & 0.71 & [29] \\
\hline Heptane & $280-507$ & $280-507$ & 29 & 29 & -0.66819 & 3.65474 & -2.57040 & 0.24 & 1.23 & [29] \\
\hline 2-Methylhexane & $284-524$ & $284-512$ & 41 & 40 & -0.75177 & 3.62326 & -2.77636 & 0.53 & 1.03 & [29] \\
\hline 3-Methylhexane & $283-529$ & $288-523$ & 44 & 42 & -0.79082 & 3.39884 & -2.42917 & 0.68 & 0.82 & [29] \\
\hline 3-Ethylpentane & $300-532$ & $300-523$ & 28 & 27 & -0.83184 & 3.69644 & -3.06484 & 0.43 & 1.15 & [29] \\
\hline 2,2-Dimethylpentane & $286-512$ & $286-503$ & 28 & 27 & -0.93326 & 3.47375 & -2.96623 & 0.43 & 0.66 & [29] \\
\hline 2,3-Dimethylpentane & $260-529$ & $297-522$ & 37 & 31 & -0.90001 & 3.49629 & -2.85731 & 0.94 & 1.00 & [29] \\
\hline 2,2,3-Trimethylbutane & $290-525$ & $273-513$ & 41 & 42 & -1.06114 & 3.19238 & -2.86120 & 0.23 & 0.53 & [29] \\
\hline Octane & $312-544$ & $312-544$ & 30 & 30 & -0.18867 & 3.76582 & -1.56481 & 0.66 & 1.04 & [29] \\
\hline Nonane & $333-503$ & $313-423$ & 34 & 12 & 0.16220 & 3.41296 & 0.63763 & 0.92 & 0.83 & [28] \\
\hline$n$-Decane & $353-523$ & $333-443$ & 33 & 12 & 0.41578 & 3.07020 & 2.86173 & 1.54 & 0.53 & [28] \\
\hline$n$-Undecane & $371-498$ & $353-473$ & 28 & 13 & 0.54595 & 2.65026 & 5.14323 & 1.72 & 0.68 & [28] \\
\hline$n$-Dodecane & $405-510$ & $373-483$ & 24 & 12 & 0.52746 & 2.12014 & 6.96426 & 1.50 & 0.86 & [28] \\
\hline$n$-Tridecane & $422-529$ & $393-513$ & 24 & 13 & 0.45078 & 1.75135 & 7.71931 & 1.80 & 1.04 & [28] \\
\hline$n$-Tetradecane & $438-548$ & $413-523$ & 24 & 12 & 0.24487 & 1.58747 & 6.09753 & 1.53 & 0.73 & [28] \\
\hline$n$-Pentadecane & $409-577$ & $423-543$ & 30 & 13 & -0.20370 & 1.35564 & 2.79036 & 1.59 & 0.42 & [28] \\
\hline$n$-Hexadecane & $462-573$ & $443-553$ & 24 & 12 & -0.22827 & 2.32076 & -4.39664 & 1.91 & 1.01 & [28] \\
\hline Ethene & $120-281$ & $120-277$ & 41 & 40 & 1.22085 & 1.76200 & -1.58983 & 1.68 & 4.10 & [29] \\
\hline Propene & $163-364$ & $188-364$ & 33 & 29 & 0.50218 & 2.47508 & -2.68570 & 0.98 & 2.57 & [29] \\
\hline 1-Butene & $215-413$ & $215-388$ & 40 & 35 & -0.52298 & 2.81798 & -3.33942 & 0.19 & 1.99 & [29] \\
\hline 1-Pentene & $221-463$ & $221-443$ & 37 & 34 & -1.17322 & 3.14751 & -3.88858 & 0.21 & 0.43 & [29] \\
\hline 1-Hexene & $283-353$ & $283-334$ & 45 & 33 & -1.23842 & 3.36936 & -3.63016 & 0.09 & 0.03 & [29] \\
\hline Benzene & $284-550$ & $284-550$ & 42 & 42 & -1.06972 & 2.94098 & -3.54694 & 0.20 & 0.51 & [29] \\
\hline Ethylbenzene & $297-616$ & $297-487$ & 33 & 20 & -0.86490 & 3.30361 & -2.34217 & 0.82 & 0.53 & [29] \\
\hline Toluene & $282-546$ & $282-546$ & 29 & 29 & -1.15521 & 3.08131 & -2.78948 & 0.16 & 1.15 & [29] \\
\hline
\end{tabular}


Table 1 (continued)

\begin{tabular}{|c|c|c|c|c|c|c|c|c|c|c|}
\hline \multirow[t]{2}{*}{ Compound } & \multicolumn{2}{|c|}{ Temp. Range (K) } & \multicolumn{2}{|c|}{ No. of data pts. } & \multirow[t]{2}{*}{$C_{1}$} & \multirow[t]{2}{*}{$C_{2}$} & \multirow[t]{2}{*}{$C_{3}$} & \multicolumn{2}{|c|}{$\operatorname{AAD}(\%)$} & \multirow[t]{2}{*}{ Data ref. } \\
\hline & $P^{\mathrm{V}}$ & $V^{\mathrm{L}}$ & $\overline{P^{V}}$ & $\overline{V^{\mathrm{L}}}$ & & & & $P^{\mathrm{V}}$ & $V^{L}$ & \\
\hline$o$-Xylene & $303-630$ & $303-612$ & 38 & 36 & -0.80773 & 3.35803 & -2.15722 & 0.58 & 1.34 & [29] \\
\hline$m$-Xylene & $302-616$ & $302-582$ & 37 & 33 & -0.76506 & 3.25647 & -1.94749 & 0.88 & 0.69 & [29] \\
\hline$p$-Xylene & $296-616$ & $296-491$ & 42 & 26 & -0.79954 & 3.19317 & -1.87191 & 0.64 & 1.01 & [29] \\
\hline Cyclopropane & $174-397$ & $197-386$ & 40 & 34 & 0.71588 & 2.07302 & -1.97358 & 1.71 & 1.88 & [29] \\
\hline Cyclobutane & $203-285$ & $203-273$ & 41 & 35 & -0.33404 & 2.58199 & -2.94394 & 0.32 & 1.62 & [29] \\
\hline Cyclopentane & $229-510$ & $229-503$ & 40 & 39 & -0.79429 & 2.89539 & -3.48003 & 0.12 & 1.22 & [29] \\
\hline Cyclohexane & $279-491$ & $279-491$ & 35 & 35 & -1.12371 & 3.16102 & -3.79000 & 0.13 & 0.52 & [29] \\
\hline Cycloheptane & $283-604$ & $290-575$ & 45 & 40 & -1.12610 & 3.26814 & -3.21087 & 0.69 & 0.68 & [29] \\
\hline Propyne & $217-401$ & $217-387$ & 40 & 37 & -0.14506 & 2.97974 & -3.55069 & 0.31 & 1.69 & [29] \\
\hline 1-Butyne & $202-282$ & $242-282$ & 41 & 21 & 1.34082 & 1.53164 & -2.07124 & 0.34 & 0.11 & [29] \\
\hline Methanol & $227-511$ & $220-482$ & 39 & 36 & -1.73089 & 4.55337 & -7.42214 & 1.02 & 0.96 & [29] \\
\hline Ethanol & $231-511$ & $231-503$ & 36 & 35 & -1.20047 & 7.36221 & -13.2154 & 1.83 & 1.07 & [29] \\
\hline 1-Propanol & $280-525$ & $280-517$ & 30 & 29 & -0.62505 & 8.21378 & -13.9506 & 1.38 & 0.84 & [29] \\
\hline 2-Propanol & $279-500$ & $230-494$ & 33 & 39 & -0.48940 & 8.56535 & -15.6307 & 0.95 & 1.20 & [29] \\
\hline 1-Butanol & $295-556$ & $295-548$ & 32 & 31 & -0.25057 & 8.00124 & -12.9622 & 0.69 & 1.83 & [29] \\
\hline 2-Butanol & $281-525$ & $281-488$ & 34 & 29 & -0.31623 & 7.17247 & -11.7186 & 0.49 & 2.61 & [29] \\
\hline $\begin{array}{l}\text { 2-Methyl-1- } \\
\text { propanol }\end{array}$ & $300-543$ & $300-535$ & 29 & 28 & -0.25259 & 7.82728 & -12.7573 & 0.97 & 2.01 & [29] \\
\hline $\begin{array}{l}\text { 2-Methyl-2- } \\
\text { propanol }\end{array}$ & $304-443$ & $304-443$ & 35 & 35 & -0.18159 & 8.45516 & -14.5657 & 0.45 & 2.16 & [29] \\
\hline 1-Pentanol & $278-508$ & $278-508$ & 29 & 29 & 0.12884 & 7.60194 & -11.0150 & 0.18 & 1.12 & [29] \\
\hline 1-Hexanol & $310-428$ & $310-428$ & 17 & 17 & 0.66938 & 10.8773 & -12.9516 & 0.28 & 1.32 & [29] \\
\hline 1-Heptanol & $351-445$ & $351-445$ & 13 & 13 & 0.60239 & 6.42386 & -6.55448 & 0.40 & 0.35 & [29] \\
\hline 1-Octanol & $364-549$ & $364-549$ & 23 & 23 & 0.77058 & 5.76596 & -4.06642 & 0.90 & 0.13 & [29] \\
\hline 1-Nonanol & $366-481$ & $366-481$ & 15 & 15 & 0.82941 & 4.94632 & -1.47716 & 0.26 & 0.05 & [29] \\
\hline 1-Decanol & $327-526$ & $327-526$ & 24 & 24 & 0.85601 & 4.35481 & -0.43164 & 0.46 & 0.41 & [29] \\
\hline Acetone & $253-508$ & $253-493$ & 35 & 33 & -1.49247 & 2.54349 & -3.10093 & 0.97 & 2.64 & [29] \\
\hline 2-Butanone & $272-536$ & $272-319$ & 35 & 7 & -1.40259 & 3.30402 & -3.87178 & 0.06 & 0.15 & [29] \\
\hline 2-Pentanone & $282-353$ & $282-353$ & 11 & 11 & -1.05362 & 3.09769 & -2.60213 & 0.07 & 0.20 & [29] \\
\hline 3-Pentanone & $282-353$ & $282-353$ & 11 & 11 & -1.11238 & 3.91647 & -4.29502 & 1.12 & 0.05 & [29] \\
\hline $\begin{array}{l}\text { 3-Methyl-2- } \\
\text { butanone }\end{array}$ & $276-549$ & $288-319$ & 45 & 6 & -1.08925 & 3.64100 & -3.55369 & 0.60 & 0.02 & [29] \\
\hline 2-Hexanone & $299-427$ & $299-332$ & 40 & 11 & -0.56396 & 3.80167 & -2.67369 & 0.04 & 0.31 & [29] \\
\hline 3-Hexanone & $299-407$ & $297-324$ & 44 & 12 & -0.63368 & 3.74362 & -2.73628 & 0.07 & 0.20 & [29] \\
\hline $\begin{array}{l}\text { 3,3-Dimethyl-2- } \\
\text { butanone }\end{array}$ & $284-405$ & $295-353$ & 45 & 22 & -0.82022 & 3.92052 & -3.27888 & 0.06 & 0.45 & [29] \\
\hline 2-Heptanone & $274-452$ & $310-428$ & 45 & 30 & 0.33118 & 5.29518 & -2.25655 & 0.80 & 3.37 & [29] \\
\hline 5-Nonanone & $319-453$ & $301-356$ & 30 & 13 & 0.27963 & 2.19627 & 4.48415 & 2.82 & 0.41 & [29] \\
\hline Propylene oxide & $361-478$ & - & 22 & - & -3.15125 & -1.79198 & -0.20938 & 0.26 & - & [19] \\
\hline Diethyl ether & $250-467$ & $293-370$ & 59 & 8 & -1.30277 & 3.47539 & -4.15299 & 0.48 & 0.10 & {$[14,22]$} \\
\hline $\begin{array}{l}\text { Methyl propyl } \\
\text { ether }\end{array}$ & $254-333$ & - & 22 & - & -1.11464 & 3.68290 & -3.90947 & 0.08 & - & {$[15]$} \\
\hline $\begin{array}{l}\text { Isopropyl methyl } \\
\text { ether }\end{array}$ & $1250-325$ & - & 20 & - & -1.50462 & 2.76848 & -3.61444 & 0.04 & - & {$[15]$} \\
\hline $\begin{array}{l}\text { Butyl methyl } \\
\text { ether }\end{array}$ & $266-367$ & - & 25 & - & -1.19490 & 3.27202 & -3.25247 & 0.07 & - & {$[15]$} \\
\hline
\end{tabular}


Table 1 (continued)

\begin{tabular}{|c|c|c|c|c|c|c|c|c|c|c|}
\hline \multirow[t]{2}{*}{ Compound } & \multicolumn{2}{|c|}{ Temp. Range (K) } & \multicolumn{2}{|c|}{ No. of data pts. } & \multirow[t]{2}{*}{$C_{1}$} & \multirow[t]{2}{*}{$C_{2}$} & \multirow[t]{2}{*}{$C_{3}$} & \multicolumn{2}{|c|}{$\operatorname{AAD}(\%)$} & \multirow[t]{2}{*}{ Data ref } \\
\hline & $\overline{P^{\mathrm{V}}}$ & $V^{\mathrm{L}}$ & $\overline{P^{\mathrm{V}}}$ & $V^{\mathrm{L}}$ & & & & $\overline{P^{\mathrm{V}}}$ & $V^{\mathrm{L}}$ & \\
\hline $\begin{array}{l}t \text {-Butyl methyl } \\
\text { ether }\end{array}$ & $288-351$ & - & 16 & - & -2.08811 & 1.31380 & -2.77846 & 0.05 & - & {$[15]$} \\
\hline $\begin{array}{l}\text { Ethyl propyl } \\
\text { ether }\end{array}$ & $261-359$ & - & 26 & - & -0.42389 & 5.49225 & -4.39952 & 0.01 & - & {$[15]$} \\
\hline Dipropyl ether & $293-388$ & - & 23 & - & -0.39928 & 4.14864 & -2.40607 & 0.007 & - & {$[15]$} \\
\hline $\begin{array}{l}\text { Methyl phenyl } \\
\text { ether }\end{array}$ & $383-437$ & - & 17 & - & -0.87186 & 4.31379 & -4.31540 & 0.004 & - & {$[15]$} \\
\hline $\begin{array}{l}\text { Ethyl phenyl } \\
\text { ether }\end{array}$ & $391-454$ & - & 18 & - & -1.54123 & 0.30709 & -0.01433 & 0.005 & - & [15] \\
\hline Ethanoic acid & $304-415$ & $293-490$ & 27 & 14 & -1.59208 & 2.20598 & -2.69563 & 0.96 & 2.49 & {$[22,16]$} \\
\hline Propanoic acid & $328-438$ & $288-490$ & 28 & 15 & -0.63879 & 4.76718 & -6.20723 & 0.91 & 2.44 & {$[22,17]$} \\
\hline Butyric acid & $350-452$ & - & 26 & - & 2.81838 & 22.5235 & -19.6192 & 0.06 & - & {$[17]$} \\
\hline Benzene acid & $405-523$ & $403-453$ & 8 & 6 & 0.55604 & 5.38674 & -5.07857 & 1.10 & 0.61 & {$[30]$} \\
\hline Methyl formate & $294-305$ & - & 10 & - & -0.67765 & 3.42796 & -1.91868 & 0.80 & - & {$[20]$} \\
\hline Ethyl formate & $277-327$ & - & 13 & - & -1.36645 & 3.30125 & -4.08239 & 0.05 & - & [20] \\
\hline Propyl formate & $299-355$ & - & 16 & - & -1.55669 & 2.60912 & -3.47168 & 0.46 & - & {$[20]$} \\
\hline Methyl acetate & $260-351$ & - & 26 & - & -1.49780 & 3.17459 & -4.03031 & 0.03 & - & [17] \\
\hline Ethyl acetate & $253-523$ & $273-503$ & 58 & 24 & -0.95984 & 3.64834 & -3.45716 & 0.28 & 0.76 & {$[30,17]$} \\
\hline Propyl acetate & $290-399$ & $297-373$ & 28 & 10 & -0.55197 & 3.52582 & -2.25033 & 0.26 & 0.38 & {$[17,24]$} \\
\hline Butyl acetate & $326-405$ & - & 15 & - & -1.19003 & 1.51766 & -1.28465 & 0.02 & - & [24] \\
\hline Fluoro methane & $132-288$ & $144-296$ & 38 & 37 & 0.03942 & 1.76605 & -1.93107 & 0.50 & 1.35 & [29] \\
\hline $\begin{array}{l}\text { Dichloro fluoro } \\
\text { methane }\end{array}$ & $245-450$ & $207-439$ & 38 & 43 & -1.14537 & 2.87157 & -3.00653 & 0.26 & 0.56 & [29] \\
\hline Trifluoro methane & $141-298$ & $191-298$ & 45 & 31 & -1.34377 & 3.05069 & -3.66627 & 0.10 & 1.11 & [29] \\
\hline Trichloro methane & $216-431$ & $209-524$ & 31 & 45 & -1.05848 & 3.36860 & -3.13252 & 0.99 & 1.24 & [29] \\
\hline Dichloro methane & $212-503$ & $212-414$ & 40 & 28 & -1.03726 & 2.53839 & -3.17354 & 0.39 & 1.92 & [29] \\
\hline $\begin{array}{l}\text { Tetrafluoro } \\
\text { methane }\end{array}$ & $89-227$ & $92-183$ & 45 & 30 & -0.89309 & 2.85818 & -3.27113 & 0.43 & 1.03 & [29] \\
\hline $\begin{array}{l}\text { Trichloro fluoro } \\
\text { methane }\end{array}$ & $226-465$ & $180-465$ & 37 & 44 & -0.99064 & 2.70948 & -1.97476 & 0.53 & 1.07 & [29] \\
\hline $\begin{array}{l}\text { Dichloro difluoro } \\
\text { methane }\end{array}$ & $172-383$ & $151-378$ & 41 & 44 & -1.07475 & 2.75424 & -2.57806 & 0.15 & 0.90 & [29] \\
\hline Chloro methane & $193-398$ & $193-398$ & 40 & 40 & 0.31599 & 2.02707 & -2.11970 & 0.56 & 1.98 & [29] \\
\hline $\begin{array}{l}\text { Chloro difluoro } \\
\text { methane }\end{array}$ & $183-365$ & $203-365$ & 45 & 40 & -1.20197 & 2.90998 & -3.27968 & 0.25 & 0.88 & [29] \\
\hline $\begin{array}{l}\text { 1,1-Difluoro } \\
\text { ethane }\end{array}$ & $203-382$ & $232-382$ & 44 & 37 & -1.38034 & 2.62107 & -2.99642 & 1.63 & 1.06 & [29] \\
\hline $\begin{array}{l}\text { 1,2-Dichloro- } \\
\text { 1,1,2,2-tetrafluoro }\end{array}$ & $\begin{array}{l}245-413 \\
\text { ethane }\end{array}$ & $214-403$ & 34 & 38 & -0.13756 & 2.81518 & 0.85946 & 1.11 & 1.20 & [29] \\
\hline Chloro ethane & $217-460$ & $244-383$ & 45 & 26 & -0.79618 & 2.45677 & -3.02460 & 0.40 & 1.99 & [29] \\
\hline $\begin{array}{l}\text { 1,2-Dichloro } \\
\text { ethane }\end{array}$ & $279-403$ & $243-349$ & 35 & 30 & -1.16597 & 3.09077 & -2.35803 & 0.03 & 0.46 & [29] \\
\hline Fluoro ethane & $169-375$ & $173-234$ & 45 & 14 & -0.55808 & 2.39104 & -2.75868 & 0.29 & 0.19 & [29] \\
\hline $\begin{array}{l}\text { 1,1,2-Trichloro- } \\
\text { 1,2,2-trifluoro etha }\end{array}$ & $\begin{array}{l}304-482 \\
\text { ane }\end{array}$ & $281-465$ & 33 & 34 & 0.06220 & 2.71073 & 1.83349 & 1.76 & 0.84 & [29] \\
\hline $\begin{array}{l}\text { 1,1,1-Trifluoro } \\
\text { ethane }\end{array}$ & $251-371$ & $243-336$ & 43 & 33 & -1.37474 & 2.65120 & -2.88683 & 0.76 & 0.17 & [29] \\
\hline
\end{tabular}


Table 1 (continued)

\begin{tabular}{|c|c|c|c|c|c|c|c|c|c|c|}
\hline \multirow[t]{2}{*}{ Compound } & \multicolumn{2}{|c|}{ Temp. Range (K) } & \multicolumn{2}{|c|}{ No. of data pts. } & \multirow[t]{2}{*}{$C_{1}$} & \multirow[t]{2}{*}{$C_{2}$} & \multirow[t]{2}{*}{$C_{3}$} & \multicolumn{2}{|c|}{$\operatorname{AAD}(\%)$} & \multirow[t]{2}{*}{ Data ref } \\
\hline & $\overline{P^{\mathrm{V}}}$ & $V^{\mathrm{L}}$ & $\overline{P^{\mathrm{V}}}$ & $V^{\mathrm{L}}$ & & & & $\overline{P^{V}}$ & $V^{L}$ & \\
\hline $\begin{array}{l}\text { 1,1,1-Trichloro } \\
\text { ethane }\end{array}$ & $173-346$ & $200-327$ & 45 & 33 & -0.93841 & 2.56792 & -1.23895 & 0.52 & 2.80 & [29] \\
\hline $\begin{array}{l}\text { Hexafluoro } \\
\text { benzene }\end{array}$ & $283-387$ & - & 23 & - & 18.0081 & 31.5650 & 12.1060 & 1.85 & - & {$[20]$} \\
\hline Methyl amine & $190-267$ & - & 13 & - & -0.70116 & 2.84724 & -4.22465 & 0.13 & - & {$[20]$} \\
\hline Dimethyl amine & $201-280$ & - & 13 & - & -1.70652 & 4.80058 & -8.95688 & 0.05 & - & {$[20]$} \\
\hline Trimethyl amine & $193-277$ & - & 19 & - & -0.27031 & 4.27975 & -4.51601 & 0.03 & - & {$[20]$} \\
\hline Diethyl amine & $305-334$ & - & 13 & - & 1.38154 & 10.2608 & -8.24635 & 0.24 & - & {$[20]$} \\
\hline 1-Propyl amine & $296-351$ & - & 12 & - & 1.4487 & 3.84500 & -5.57313 & 0.006 & - & [20] \\
\hline 2-Propyl amine & $277-334$ & - & 13 & - & -1.42340 & 3.26901 & -4.69386 & 0.005 & - & {$[20]$} \\
\hline $\begin{array}{l}\text { 2-Methyl-2- } \\
\text { propyl amine }\end{array}$ & $292-348$ & - & 12 & - & -1.11634 & 4.37654 & -5.43046 & 0.005 & - & {$[20]$} \\
\hline $\begin{array}{l}n, n \text {-Dimethyl } \\
\text { formamide }\end{array}$ & $303-363$ & - & 7 & - & 0.88067 & 7.55181 & -5.22791 & & - & {$[20]$} \\
\hline Aniline & $331-699$ & $293-453$ & 16 & 17 & -0.72183 & 4.00981 & -3.52901 & 3.32 & 0.21 & {$[30]$} \\
\hline Acetonitrile & $439-530$ & - & 15 & - & 2.30928 & 8.67001 & -5.96392 & 1.04 & - & {$[25]$} \\
\hline Tetrahydrofuran & $296-373$ & - & 15 & - & -1.09099 & 2.63336 & -3.17383 & 0.003 & - & {$[20]$} \\
\hline Pyridine & $340-426$ & - & 15 & - & -1.29519 & 3.29104 & -4.09374 & 0.01 & - & {$[20]$} \\
\hline 2-Furfuraldehyde & $329-434$ & - & 34 & - & 3.45577 & 15.2371 & -6.59535 & 0.87 & - & {$[20]$} \\
\hline$p$-Nitro toluene & $417-512$ & - & 18 & - & 3.71841 & 6.86415 & 5.84124 & 1.48 & - & [18] \\
\hline$m$-Cresol & $407-594$ & - & 22 & - & -1.57900 & 0.75438 & -1.86520 & 0.84 & - & {$[20,26]$} \\
\hline$o$-Cresol & $393-464$ & - & 5 & - & 0.52861 & 5.18826 & -1.40940 & 0.22 & - & {$[20]$} \\
\hline$p$-Cresol & $401-475$ & - & 6 & - & 1.23334 & 6.79198 & -2.37839 & 0.20 & - & {$[20]$} \\
\hline Phenol & $345-456$ & $329-449$ & 35 & 38 & -0.21334 & 4.70549 & -3.49593 & 0.49 & 1.57 & [29] \\
\hline Methyl propionate & $294-352$ & - & 15 & - & 0.59544 & 7.27903 & -4.54050 & 0.09 & - & {$[20]$} \\
\hline Grand Average & & & 4409 & 3092 & & & & 0.61 & 1.19 & \\
\hline
\end{tabular}

One binary interaction parameter $k_{i j}$ was employed in the VLE calculations of fluid mixtures. The objective function used in the VLE calculations to determine the optimal binary interaction parameters was:

$$
Q^{\prime}=\frac{100}{N} \Sigma\left[\left|\frac{P^{\mathrm{cal}}-P^{\exp }}{P^{\exp }}\right|+\left|y^{\mathrm{cal}}-y^{\exp }\right|\right]
$$

\section{Results and discussion}

For each pure fluid, $\alpha, \zeta_{\mathrm{c}}$, and two temperature functions $F_{1}$ and $F_{2}$ were determined by regressing the saturated properties of pure compounds. Their values were then generalized as functions of pure fluid properties, such as the molecular weight (MW), acentric factor and the normal 
Table 2

Comparison of calculated pure fluid vapor pressures and saturated liquid molar volumes by various EOS models

\begin{tabular}{|c|c|c|c|c|c|c|}
\hline \multirow[t]{2}{*}{ EOS models } & \multicolumn{2}{|c|}{ No. of data points } & \multicolumn{2}{|c|}{$\operatorname{AADP}(\%)$} & \multicolumn{2}{|c|}{$\operatorname{AADV}^{\mathrm{L}}(\%)$} \\
\hline & $\overline{P^{\mathrm{V}}}$ & $V^{\mathrm{L}}$ & $\mathrm{A}$ & $\mathrm{B}$ & $\mathrm{A}$ & $\mathrm{B}$ \\
\hline $\operatorname{MSRK}(\mathrm{A}) / \mathrm{CPHB}(\mathrm{B})$ & 3150 & 2433 & 1.12 & 0.62 & 15.2 & 1.17 \\
\hline PRSV2 (A) /CPHB (B) & 2109 & 1340 & 0.63 & 0.68 & 6.61 & 1.23 \\
\hline $\mathrm{PT}(\mathrm{A}) / \mathrm{CPHB}(\mathrm{B})$ & 1735 & 1349 & 2.92 & 0.79 & 1.93 & 1.38 \\
\hline $\operatorname{CCOR}(\mathrm{A}) / \mathrm{CPHB}(\mathrm{B})$ & 2726 & 2121 & 2.06 & 0.73 & 1.79 & 1.27 \\
\hline
\end{tabular}

boiling temperature. Over 140 pure nonpolar and polar fluids were examined in the regression and the following generalized correlations were obtained:

$$
\alpha=1.0003-0.2719 M+3.7311 M^{2}-1.0827 M^{3}+0.1144 M^{4}-4.1276 \times 10^{-3} M^{5}
$$

where

$$
M=\frac{\mathrm{MW} \times \omega}{39.948}
$$

MW is the molecular weight and the constant 39.948 is the MW of argon. The acentric factor of argon is 0.001 and the $\alpha$ value of argon is zero, evaluated from the above equations. The physical significance of $\alpha$ is the nonspherical shape of a molecule. For fluids other than the simple spherical

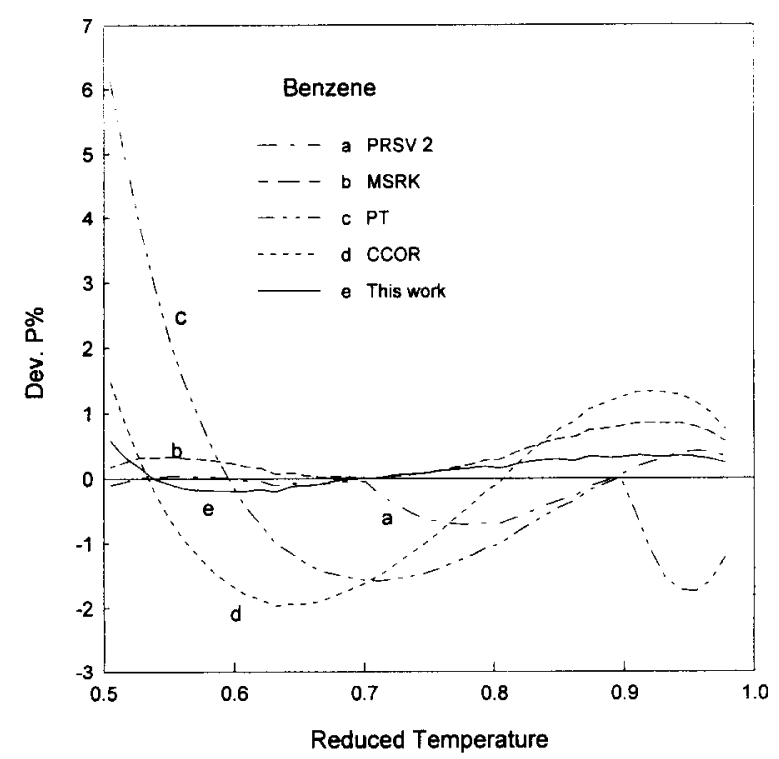

Fig. 1. Comparison of saturated vapor pressures of benzene calculated by various equations of state. 
molecules, the numerical values of $\alpha$ are always greater than unity. The temperature functions $F_{1}$ and $F_{2}$ were correlated by the following forms:

$$
\begin{aligned}
& F_{1}=\left[1+C_{1}\left(1-\sqrt{T_{R}}\right)+C_{2}\left(1-T_{R}\right)\right]^{2} \\
& F_{2}=\left[1+C_{3}\left(1-T_{R}^{2 / 3}\right)+C_{4}\left(1-T_{R}^{2 / 3}\right)^{2}+C_{5}\left(1-T_{R}^{2 / 3}\right)^{3}\right]^{2}
\end{aligned}
$$

In the last two equations, parameters $C_{2}$ and $C_{5}$ were generalized by the following functions of pure fluid properties:

$$
\begin{aligned}
& C_{2}=-1.4671+3.6889\left(\frac{T_{\mathrm{c}}}{\alpha T_{b}}\right)-2.0005\left(\frac{T_{\mathrm{c}}}{\alpha T_{b}}\right)^{2}+5.2614 \sqrt{\omega Z_{\mathrm{c}}} \\
& C_{5}=7.9885-4.3604 e^{\omega}+1.4554 \mathrm{MW} \omega^{3.063}-21.395 \alpha\left(\zeta_{\mathrm{c}}-Z_{\mathrm{c}}\right)-4.0692 Z_{\mathrm{c}} \alpha^{1.667}
\end{aligned}
$$

The critical properties were either taken from literature or estimated by group contribution method ([9]). Parameter $\zeta_{c}$ was correlated by the following equation:

$$
\zeta_{\mathrm{c}}=0.2974+0.1123 \omega-0.9585 \omega Z_{\mathrm{c}}+7.7731 \times 10^{-4} \omega \mathrm{MW}\left(\frac{T_{b}}{T_{\mathrm{c}}}\right)
$$

For the pure fluids investigated in this study, $\zeta_{\mathrm{c}}$ values are close to their critical compressibility factors $Z_{c}$. The numerical values of $\zeta_{c}$ are always between 0.215 and 0.312 . From the above correlations, it is concluded that for each pure fluid, there are three individual EOS parameters: $C_{1}$, $C_{3}$, and $C_{4}$. Table 1 lists the pure fluid parameters of the CPHB EOS, and the deviations of the calculated saturated properties. Generally, the results are satisfactory where the absolute average

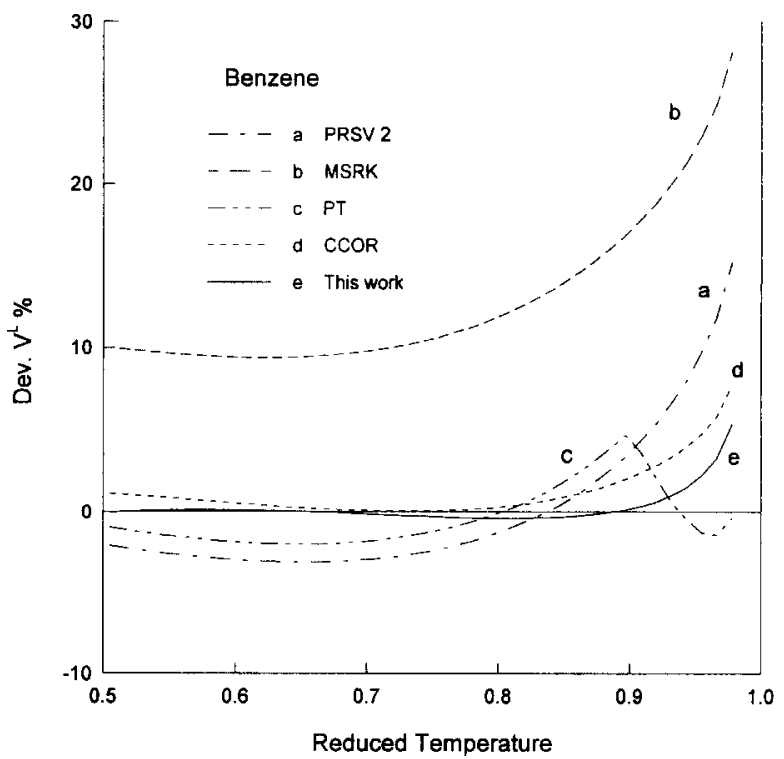

Fig. 2. Comparison of saturated liquid molar volumes of benzene calculated by various equations of state. 


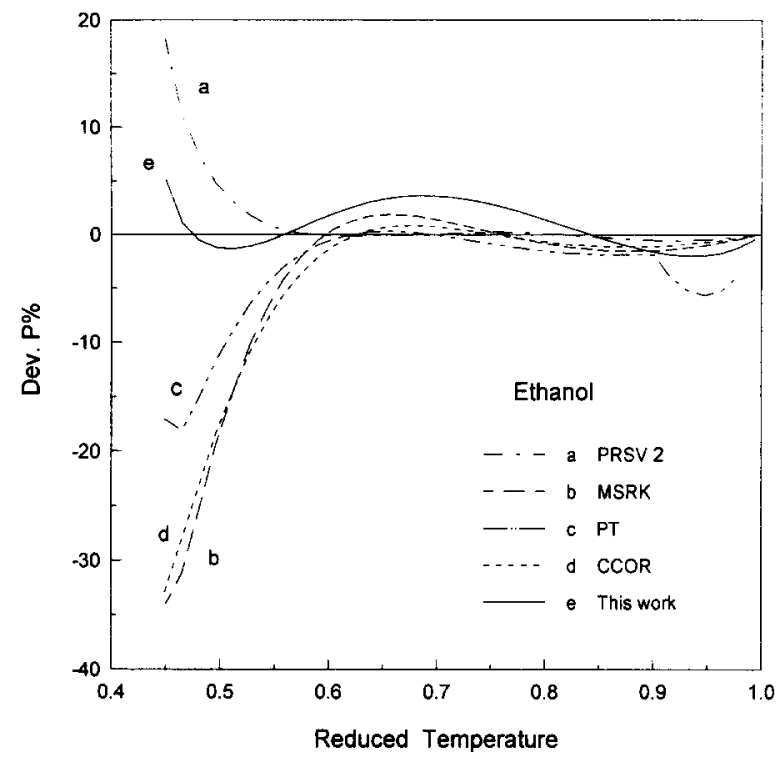

Fig. 3. Comparison of saturated vapor pressures of ethanol calculated by various equations of state.

calculated errors in vapor pressure and saturated liquid molar volume are about $1 \%$. We have compared the calculated results from the CPHB EOS with those from other EOS models (MSRK, [10]; PRSV2, [11]; CCOR, [12]; PT, [13]). There are three parameters for the PRSV2 EOS, and other EOS models have two parameters for each pure fluid. The comparison of pure fluid calculations is shown in Table 2. The CPHB EOS has shown good calculation results on both vapor pressures and

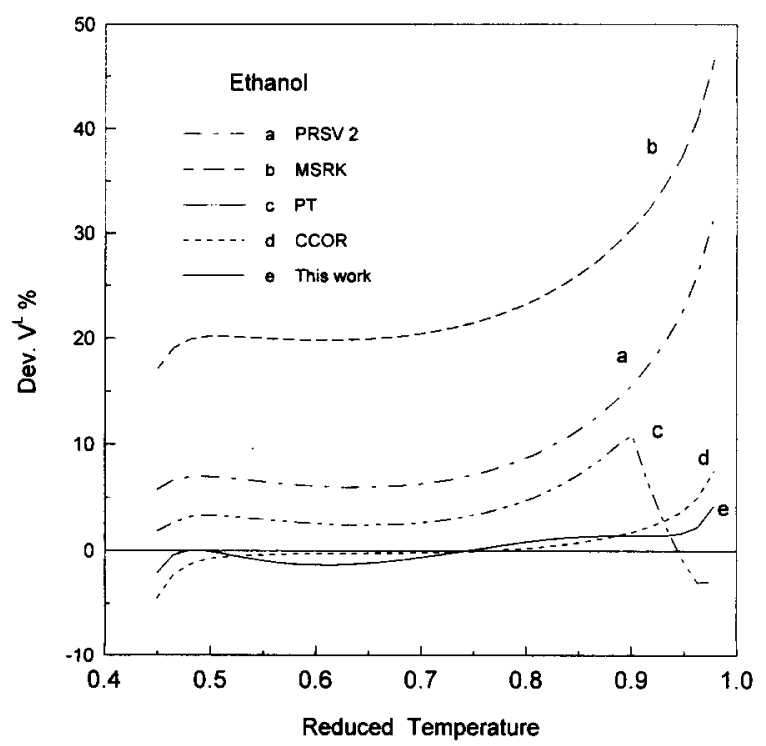

Fig. 4. Comparison of saturated liquid molar volumes of ethanol calculated by various equations of state. 


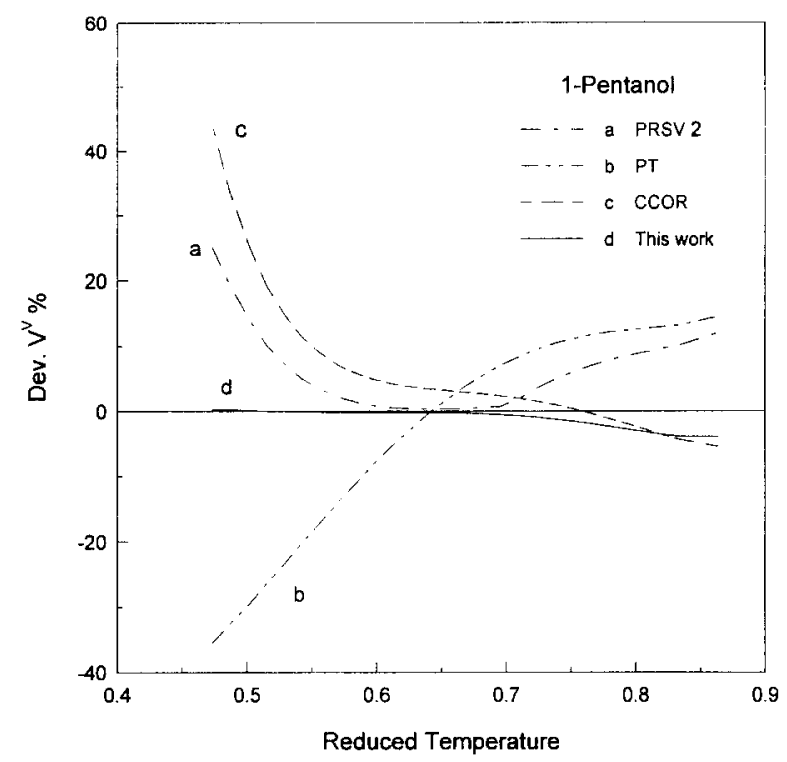

Fig. 5. Comparison of saturated vapor molar volumes of 1-pentanol calculated by various equations of state.

saturated liquid molar volumes. It is demonstrated that in over at least 1000 data points, the CPHB EOS yielded overall better results than those from other EOS models. Graphical comparisons are also presented for nonpolar and polar fluids. Figs. 1 and 2 shows the error distributions of the calculated vapor pressures and liquid molar volumes of benzene from various EOS. The CPHB EOS has the

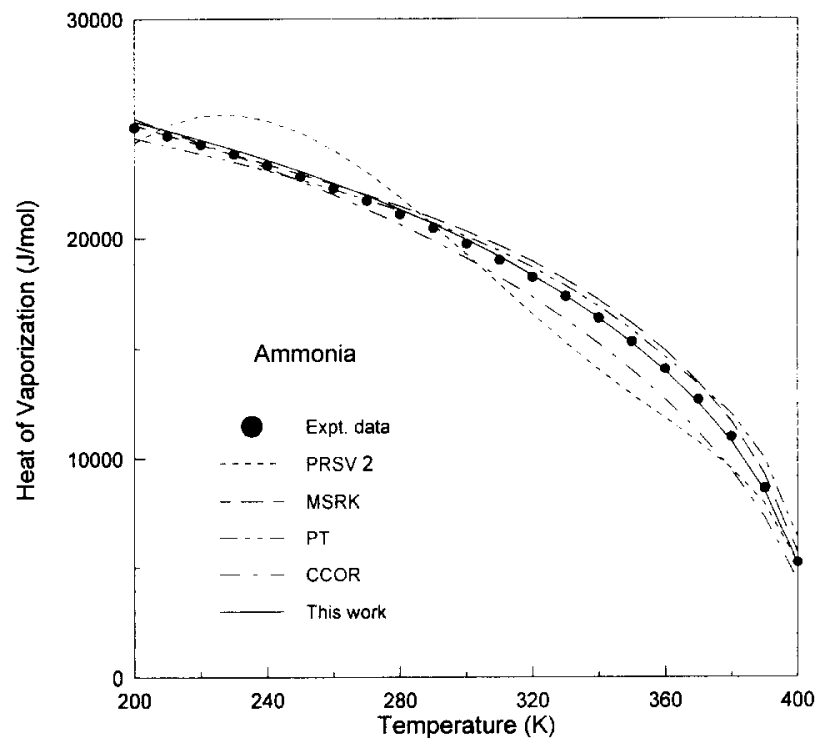

Fig. 6. Comparison of heats of vaporization of ammonia calculated by various equations of state. (Data source: Smith and Srivasta, 1986 [29]). 


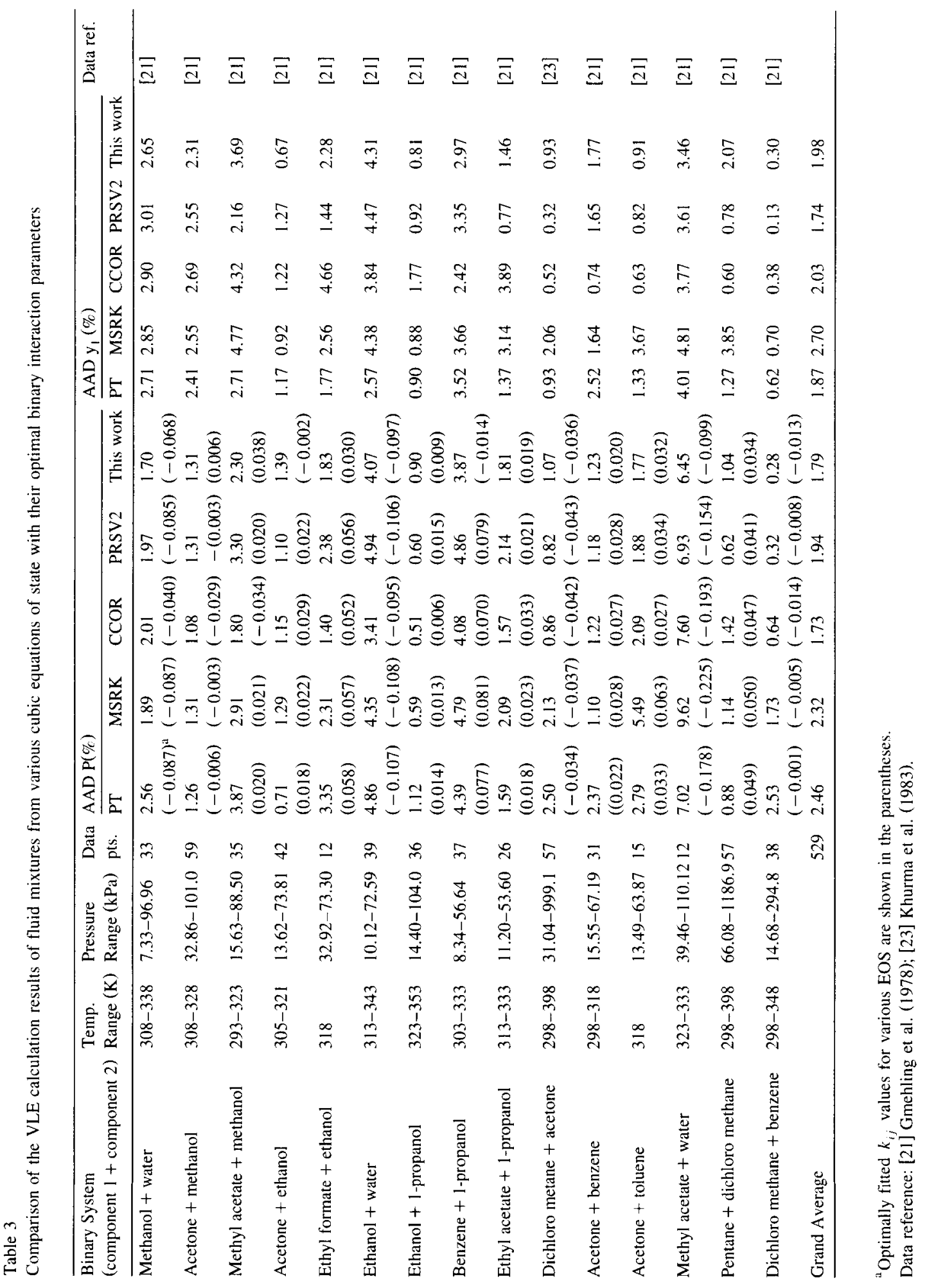


Table 4

Comparison of binary VLE calculation results using various equations of state

\begin{tabular}{|c|c|c|c|c|c|}
\hline \multirow[t]{2}{*}{ EOS models } & \multirow[t]{2}{*}{ No. of data points } & \multicolumn{2}{|c|}{$\operatorname{AADP}(\%)$} & \multicolumn{2}{|c|}{$\mathrm{AADy}_{1}(\%)$} \\
\hline & & A & B & $\overline{\mathrm{A}}$ & $\mathrm{B}$ \\
\hline MSRK (A)/CPHB (B) & 1462 & 3.67 & 3.25 & 4.18 & 3.69 \\
\hline PRSV2 (A)/CPHB (B) & 646 & 3.93 & 3.66 & 4.21 & 3.65 \\
\hline PT (A) /CPHB (B) & 630 & 3.96 & 3.69 & 4.12 & 3.64 \\
\hline $\operatorname{CCOR}(\mathrm{A}) / \mathrm{CPHB}(\mathrm{B})$ & 674 & 2.06 & 2.20 & 2.88 & 2.70 \\
\hline
\end{tabular}

smallest peak and average deviations over the entire reduced density range. Figs. 3 and 4 shows another comparison for the polar fluid of ethanol. It is demonstrated that except the CCOR and CPHB EOS, all other models have relatively larger errors in saturated liquid molar volume calculations. On the other hand, the CPHB EOS has the overall satisfactory results on vapor pressures. The deviations on saturated vapor volumes for 1-pentanol is shown in Fig. 5. It is again observed that the CPHB EOS shows superior results than those from other models. The heat of vaporization was derived from various models and the comparison for ammonia is presented in Fig. 6. It is demonstrated that the CPHB EOS has satisfactory temperature dependence and the heat of vaporization is satisfactorily determined from this EOS. The CPHB EOS gives satisfactory results on the saturated properties of pure fluids. It, however, still has some limitations beyond the saturation envelope. More efforts should be devoted to the application of this EOS to supercritical calculations.

VLE calculations were carried out for nonpolar and polar fluid mixtures. Traditional van der Waals mixing rules with one binary interaction parameter for the energy parameter of various EOS models

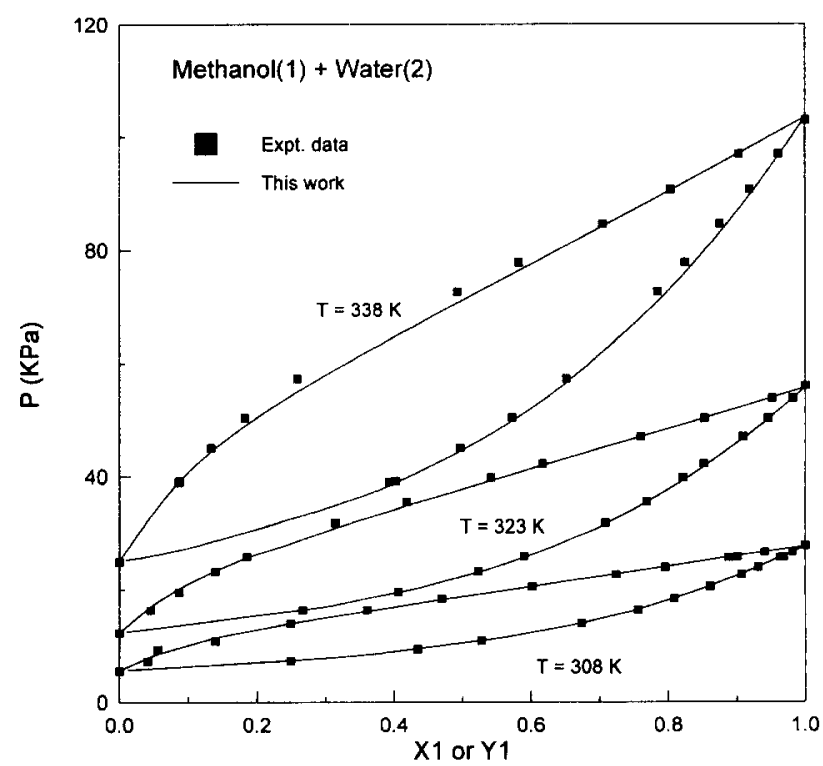

Fig. 7. Comparison of the calculated and the experimental VLE results of methanol and water mixture at various temperatures. (Data source: Gmehling et al., 1978 [21]). 


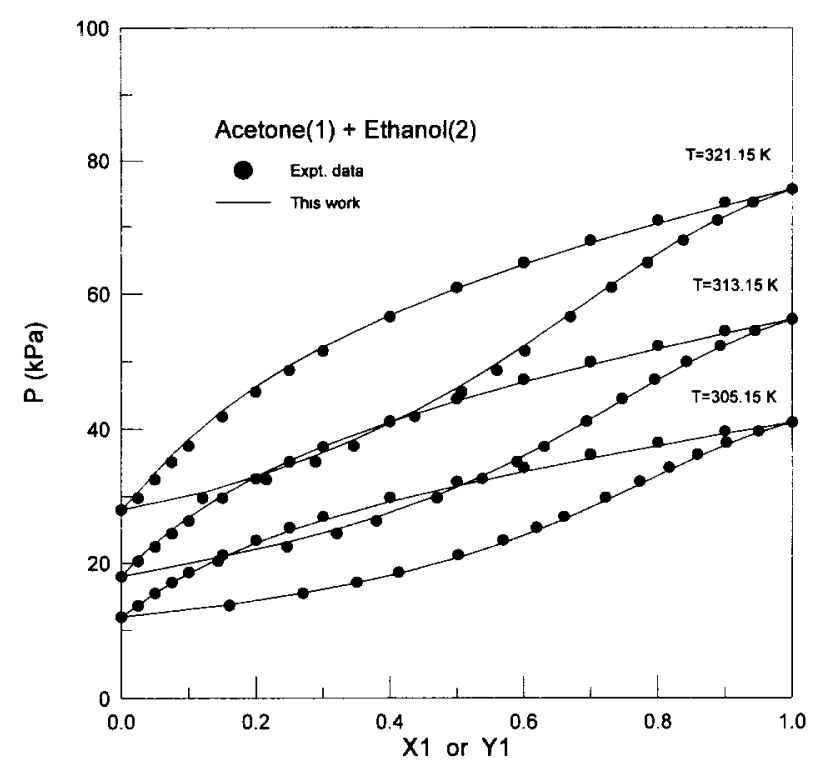

Fig. 8. Comparison of the calculated and the experimental VLE results of acetone and methanol mixtures at various temperatures. (Data source: Gmehling et al., 1978 [21]).

were employed. The calculation results and the comparison of various EOS for typical binary mixtures are shown in Table 3. All EOS models show comparable VLE calculation results with their optimally adjusted binary parameters. Table 4 shows the overall comparison of VLE calculation results. The CPHB EOS yielded slightly better deviations than those from other methods. Graphical VLE calculation results are shown in Figs. 7 and 8 for the binary mixtures of methanol + water and acetone + ethanol, respectively. It is found that the CPHB EOS gives good agreement with the experimental Pxy data at various temperatures.

\section{Conclusion}

A CPHB EOS was developed for VLE calculations of pure fluids and fluid mixtures. The repulsive part of this EOS has good physical significance and it has been shown to be in satisfactory agreement with molecular simulation data. This EOS has been extended to real fluid calculations in this study. Pure compound parameters of the CPHB EOS were determined for extensive pure nonpolar and polar fluids. The CPHB EOS yielded satisfactory results on saturated properties of pure fluids. The absolute average deviations from this EOS are better than those from other commonly used cubic EOS. VLE calculations on fluid mixtures are also successful using this EOS. The overall accuracy for mixture calculations of this EOS is also slightly better compared to those of other EOS with their optimal binary parameters.

\section{Nomenclature}

$a, b, c \quad$ Equation of state parameters

$A, B, C$ Parameters defined in Eqs. (15)-(17)

$f \quad$ Fugacity

$k \quad$ Parameter defined in Eqs. (2) and (3) 


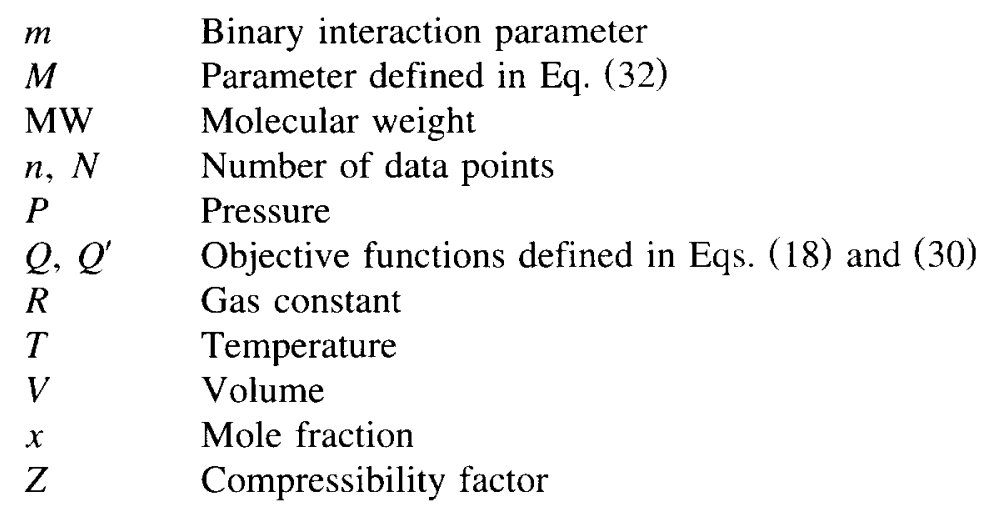

\section{Greek letters}

$\alpha \quad$ Nonspherical factor

$\zeta \quad$ Parameter defined in Eq. (6)

$\omega \quad$ Acentric factor

$\Omega \quad$ Parameter defined in Eqs. (10)-(12)

\section{Superscripts}

$\begin{array}{ll}\mathrm{L} & \text { Liquid phase } \\ \mathrm{V} & \text { Vapor phase }\end{array}$

\section{Subscripts}

$\begin{array}{ll}b & \text { Normal boiling point } \\ \mathrm{c} & \text { Critical property } \\ i, j & \text { Component } i \text { or } j \\ m & \text { Mixture parameter } \\ \mathrm{R} & \text { Reduced property }\end{array}$

\section{Acknowledgements}

The authors are grateful to the National Science Council, Republic of China for supporting this research.

\section{References}

[1] N.F. Carnahan, K.E. Starling, Equation of state for nonattracting rigid spheres, J. Chem. Phys. 51 (1969) $635-636$.

[2] H.M. Lin, H. Kim, T.M. Guo, K.C. Chao, Cubic chain-of-rotators equation of state and VLE calculations, Fluid Phase Equilib. 13 (1983) 143-152.

[3] K. Mulia, V.F. Yesavage, Development of a perturbed hard sphere equation of state for nonpolar and polar/associating fluids, Fluid Phase Equilib. 52 (1989) 67-74.

[4] L.S. Wang, T.M. Guo, A cubic simplified perturbed hard-chain equation of state for fluids with chainlike molecules, Can. J. Chem. Eng. 71 (1993) $591-604$. 
[5] T. Boublik, Hard convex body equation of state, J. Chem. Phys. 63 (1975) 4084.

[6] J.M. Walsh, K.E. Gubbins, A modified thermodynamic perturbation theory equation for molecules with fused hard sphere cores, J. Phys. Chem. 94 (1990) 5115-5120.

[7] A. Thomas, M.D. Donohue, Equation of state for chain molecules using a site-interaction model: theory, simulation and correlation of experimental data, Ind. Eng. Chem. Res. 32 (1993) 2093-2104.

[8] S.F. Chen, Y.L. Chou, Y.P. Chen, A new cubic simplified perturbed hard-body equation of state, Fluid Phase Equilib. 118 (1996) 201-219.

[9] K.M. Klincewicz, R.C. Reid, Estimation of critical properties with group contribution methods, AIChE J. 30 (1984) $137-142$.

[10] J.A. Sandarusi, A.J. Kidnay, V.F. Yesavage, Compilation of parameters for a polar fluid Soave-Redlich-Kwong equation of state, Ind. Eng. Chem. Process Des. Dev. 25 (1986) 957-963.

[11] R. Stryjek, J.H. Vera, PRSV2: A cubic equation of state for accurate vapor-liquid equilibrium correlations, Can. J. Chem. Eng. 64 (1986) 820-826.

[12] T.M. Guo, H.M. Lin, K.C. Chao, Cubic chain-of-rotators equation of state: 3. mixtures of polar substances, Ind. Eng. Chem. Process Des. Dev. 24 (1985) 768-773.

[13] N.C. Patel, A.S. Teja, A new cubic equation of state for fluids and fluid mixtures, Chem. Eng. Sci. 37 (1982) $463-473$.

[14] D. Ambrose, C.H.S. Sprake, R. Townsend, Thermodynamic properties of organic oxygen compounds: XXIX. The vapor pressures of diethyl ether, J. Chem. Thermodynamics 4 (1972) 247-254.

[15] D. Ambrose, J.H. Ellender, C.H.S. Sprake, R. Townsend, Thermodynamic properties of organic oxygen compounds: XLII. Vapor pressure of some ethers, J. Chem. Thermodynamics 8 (1976) 165-178.

[16] D. Ambrose, J.H. Ellender, C.H.S. Sprake, R. Townsend, Thermodynamic properties of organic oxygen compounds: XLV. The vapor pressure of acetic acid, J. Chem. Thermodynamics 9 (1977) 735-741.

[17] D. Ambrose, J.H. Ellender, H.A. Gundry, D.A. Lee, R. Townsend, Thermodynamic properties of organic oxygen compounds: LI. The vapor pressure of some ethers and fatty acids, J. Chem. Thermodynamics 13 (1981) 795-802.

[18] D. Ambrose, H.A. Gundry, The vapor pressure of p-nitrotoluene, J. Chem. Thermodynamics 12 (1980) 559-561.

[19] T.R. Bott, H.N. Sadler, Vapor pressure of propylene oxide, J. Chem. Eng. Data 11 (1966) 25-30.

[20] T. Boublik, V. Fried, E. Hala, The vapor pressures of pure substances, Elsevier, Amsterdam, 1973.

[21] J. Gmehling, U. Onken, W. Arlt, P. Grenzheuser, U. Weidlich, B. Kolbe, J. Rarey-Nies, Vapor-Liquid Equilibrium Data Collection, DECHEMA, Frankfurt, Germany, 1978.

[22] J.L. Hales, H.A. Gundry, J.H. Ellender, Liquid densities from 288 to $490 \mathrm{~K}$ of four organic oxygen compounds, J. Chem. Thermodynamics 15 (1983) 211-215.

[23] J.R. Khurma, O. Muthu, S. Munjal, B.D. Smith, Total-pressure vapor-liquid equilibrium data for binary systems of dichloromethane with pentane, acetone, methanol and acetonitrile, J. Chem. Eng. Data 28 (1983) 412-419.

[24] E.F. Meyer, M.J. Awe, Cohesive energies in polar organic liquids: 4. $n$-alkyl acetates, J. Chem. Eng. Data 25 (1980) $371-374$.

[25] A.H.N. Mousa, Vapor pressure and saturated vapor volume of acetonitrile, J. Chem. Thermodynamics 13 (1981) 201-202.

[26] P. Nasir, S.C. Hwang, R. Kobayashi, Development of an apparatus to measure vapor pressures at high pressures and its application to three higher-boiling compounds, Am. Chem. Soc. 25 (1980) 298-301.

[27] D.P.M. Nunes, L.A.K. Staveley, The equation of state and thermodynamic properties of liquid hydrogen chloride, J. Chem. Thermodynamics 13 (1981) 179-186.

[28] F.D. Rossini, K.S. Pitzer, R.L. Arnett, R.M. Braun, G.C. Pimental, Selected values of properties of hydrocarbons and related compounds. API Project 44, Carnegie, Pittsburgh, 1952.

[29] B.D. Smith, R. Srivastava, Thermodynamic data for pure compounds, Elsevier, Amsterdam, 1986.

[30] N.B. Vargaftik, Tables on the thermodynamic properties of liquids and gases, Hemisphere, Washington, DC, 1975. 